\title{
Some inequalities via fractional conformable integral operators
}

\author{
Kottakkaran Sooppy Nisar ${ }^{1}$, Asifa Tassaddiq2 ${ }^{2 *}$, Gauhar Rahman ${ }^{3}$ and Aftab Khan ${ }^{3}$
}

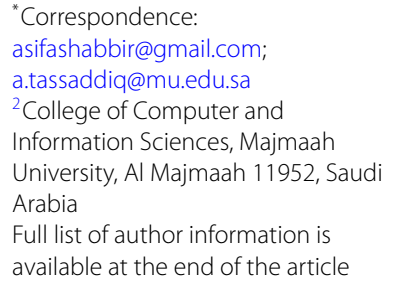

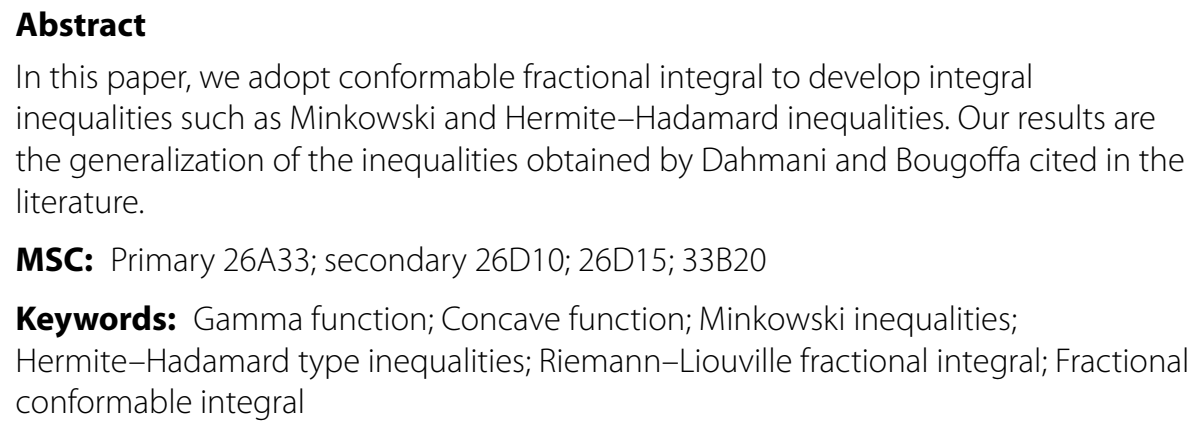
inequalities such as Minkowski and Hermite-Hadamard inequalities. Our results are the generalization of the inequalities obtained by Dahmani and Bougoffa cited in the literature.

MSC: Primary 26A33; secondary 26D10; 26D15; 33B20

Keywords: Gamma function; Concave function; Minkowski inequalities; Hermite-Hadamard type inequalities; Riemann-Liouville fractional integral; Fractional conformable integral

\section{Introduction}

The theory of fractional integral inequalities plays a vital role in the field of mathematical sciences. There is one of the most famous inequalities for convex functions known as Hermite-Hadamard inequality. Many researchers studied this inequality and published various generalizations and extensions by using fractional integral. We begin with the Hermite-Hadamard inequality, which is defined as follows: Let $f: I \subseteq \mathbb{R} \rightarrow \mathbb{R}$ be a convex function and $a, b \in I$ with $a<b$, then

$$
f\left(\frac{a+b}{2}\right) \leq \frac{1}{b-a} \int_{a}^{b} f(x) d x \leq \frac{f(a)+f(b)}{2} .
$$

Further generalizations and extensions can be found in, e.g., [3, 8, 10, 17]. In [15], the Riemann-Liouville fractional integrals $\mathfrak{I}_{a^{+}}^{\alpha}$ and $\mathfrak{I}_{b^{-}}^{\alpha}$ of order $\alpha>0$ are defined respectively by

$$
\mathfrak{I}_{a^{+}}^{\alpha} f(x)=\frac{1}{\Gamma(\alpha)} \int_{a}^{x}(x-t)^{\alpha-1} f(t) d t \quad(x>a, \Re(\alpha)>0)
$$

and

$$
\mathfrak{I}_{b^{-}}^{\alpha} f(x)=\frac{1}{\Gamma(\alpha)} \int_{x}^{b}(t-x)^{\alpha-1} f(t) d t \quad(x<b, \Re(\alpha)>0),
$$

(c) The Author(s) 2019. This article is distributed under the terms of the Creative Commons Attribution 4.0 International License (http://creativecommons.org/licenses/by/4.0/), which permits unrestricted use, distribution, and reproduction in any medium, provided you give appropriate credit to the original author(s) and the source, provide a link to the Creative Commons license, and indicate if changes were made. 
where $\Gamma$ is the gamma function (see [18]). In [7], the left- and right-sided fractional conformable integral operators are respectively defined by

$$
{ }^{\beta} \mathfrak{I}_{a^{+}}^{\alpha} f(x)=\frac{1}{\Gamma(\beta)} \int_{a}^{x}\left(\frac{(x-a)^{\alpha}-(t-a)^{\alpha}}{\alpha}\right)^{\beta-1} \frac{f(t)}{(t-a)^{1-\alpha}} d t, \quad x>a
$$

and

$$
{ }^{\beta} \mathfrak{I}_{b-}^{\alpha} f(x)=\frac{1}{\Gamma(\beta)} \int_{x}^{b}\left(\frac{(b-x)^{\alpha}-(b-t)^{\alpha}}{\alpha}\right)^{\beta-1} \frac{f(t)}{(b-t)^{1-\alpha}} d t, \quad x<b,
$$

where $\beta \in \mathbb{C}$ and $\Re(\beta)>0$. Obviously, if we consider $a=0, b=0$, and $\alpha=1$ in (4) and (5), then we get the Riemann-Liouville fractional integrals (2) and (3) respectively. In [16], Set et al. defined the following one-sided conformable fractional integral operator:

$$
{ }^{\beta} \mathfrak{I}^{\alpha} f(x)=\frac{1}{\Gamma(\beta)} \int_{0}^{x}\left(\frac{x^{\alpha}-\tau^{\alpha}}{\alpha}\right)^{\beta-1} \frac{f(\tau)}{\tau^{1-\alpha}} d \tau
$$

Recently Rahman et al. $[13,14]$ established some new inequalities of the Grüss type and certain Chebyshev-type inequalities for conformable fractional integrals. In $[5,9,11,12]$, various researchers established generalized $k$-fractional conformable integral inequalities, Minkowski and Chebyshev type integral inequalities involving generalized $k$-fractional conformable integrals. The Hermite-Hadamard type inequalities for $k$-fractional conformable integrals are found in [6]. A significant contribution by Guessab and Schmeisser [4] is an investigation of sharp integral inequalities of the Hermite-Hadamard type.

The paper is arranged as follows: In Sect. 2, the main results, which are reverse Minkowski and related Hermite-Hadamard type integral inequalities, are established by employing fractional conformable integral operators. The concluding remarks are given in Sect. 3.

\section{Main results}

In this section, we use fractional conformable integral operator to develop reverse Minkowski and Hermite-Hadamard integral inequalities. The reverse Minkowski fractional integral inequality is presented in the following theorems.

Theorem 2.1 Let $\beta, \alpha>0, \sigma \geq 1$, and let $\Phi, \Psi$ be two positive functions on $[0, \infty)$ such that, for all $x>0,{ }^{\beta} \mathfrak{I}^{\alpha} \Phi^{\sigma}(x)<\infty,{ }^{\beta} \mathfrak{I}^{\alpha} \Psi^{\sigma}(x)<\infty$. If $0<m \leq \frac{\Phi(t)}{\Psi(t)} \leq M, t \in[0, x]$, then the following inequality holds:

$$
\left({ }^{\beta} \mathfrak{I}^{\alpha} \Phi^{\sigma}(x)\right)^{\frac{1}{\sigma}}+\left({ }^{\beta} \widetilde{I}^{\alpha} \Psi^{\sigma}(x)\right)^{\frac{1}{\sigma}} \leq \frac{1+M(m+2)}{(m+1)(M+1)}\left({ }^{\beta} \mathfrak{I}^{\alpha}(\Phi+\Psi)^{\sigma}(x)\right)^{\frac{1}{\sigma}} .
$$

Proof Using the condition $\frac{\Phi(t)}{\Psi(t)}<M, t \in[0, x], x>0$, we have

$$
(M+1)^{\sigma} \Phi^{\sigma}(t) \leq M^{\sigma}(\Phi+\Psi)^{\sigma}(t) .
$$


Multiplying both sides of (8) by $\frac{1}{\Gamma(\beta)}\left(\frac{x^{\alpha}-t^{\alpha}}{\alpha}\right)^{\beta-1} t^{\alpha-1}$ and integrating the resultant inequality with respect to $t$ from 0 to $x$, we have

$$
\frac{(M+1)^{\sigma}}{\Gamma(\beta)} \int_{0}^{x}\left(\frac{x^{\alpha}-t^{\alpha}}{\alpha}\right)^{\beta-1} t^{\alpha-1} \Phi^{\sigma}(t) d t \leq \frac{M^{\sigma}}{\Gamma(\beta)} \int_{0}^{x}\left(\frac{x^{\alpha}-t^{\alpha}}{\alpha}\right)^{\beta-1} t^{\alpha-1}(\Phi+\Psi)^{\sigma}(t) d t,
$$

which can be written as

$$
{ }^{\beta} \mathfrak{I}^{\alpha} \Phi^{\sigma}(x) \leq{\frac{M^{\sigma}}{(M+1)^{\sigma}}}^{\beta} \mathfrak{I}^{\alpha}(\Phi+\Psi)^{\sigma}(x) .
$$

Hence, it follows that

$$
\left({ }^{\beta} \mathfrak{I}^{\alpha} \Phi^{\sigma}(x)\right)^{\frac{1}{\sigma}} \leq \frac{M}{(M+1)}\left({ }^{\beta} \mathfrak{I}^{\alpha}(\Phi+\Psi)^{\sigma}(x)\right)^{\frac{1}{\sigma}} .
$$

Now, using the condition $m \Psi(t) \leq \Phi(t)$, we have

$$
\left(1+\frac{1}{m}\right) \Psi(t) \leq \frac{1}{m}(\Phi(t)+\Psi(t))
$$

which yields

$$
\left(1+\frac{1}{m}\right)^{\sigma} \Psi^{\sigma}(t) \leq\left(\frac{1}{m}\right)^{\sigma}(\Phi(t)+\Psi(t))^{\sigma} .
$$

Multiplying both sides of (10) by $\frac{1}{\Gamma(\beta)}\left(\frac{x^{\alpha}-t^{\alpha}}{\alpha}\right)^{\beta-1} t^{\alpha-1}$ and integrating the resultant inequality with respect to $t$ from 0 to $x$, we get

$$
\left({ }^{\beta} \mathfrak{I}^{\alpha} \Psi^{\sigma}(x)\right)^{\frac{1}{\sigma}} \leq \frac{1}{(m+1)}\left({ }^{\beta} \mathfrak{I}^{\alpha}(\Phi+\Psi)^{\sigma}(x)\right)^{\frac{1}{\sigma}} .
$$

Thus, adding inequalities (9) and (11) yields the desired inequality.

Theorem 2.2 Let $\beta, \alpha>0, \beta \in \mathbb{C}, \sigma \geq 1$, and let $\Phi, \Psi$ be two positive functions on $[0, \infty)$ such that, for all $x>0,{ }^{\beta} \mathfrak{I}^{\alpha} \Phi^{\sigma}(x)<\infty,{ }^{\beta} \mathfrak{I}^{\alpha} \Psi^{\sigma}(x)<\infty$. If $0<m \leq \frac{\Phi(t)}{\Psi(t)} \leq M, t \in[0, x]$, then the following inequality holds:

$$
\begin{aligned}
& \left({ }^{\beta} \mathfrak{I}^{\alpha} \Phi^{\sigma}(x)\right)^{\frac{2}{\sigma}}+\left({ }^{\beta} \mathfrak{I}^{\alpha} \Psi^{\sigma}(x)\right)^{\frac{2}{\sigma}} \\
& \quad \geq\left(\frac{(M+1)(m+1)}{M}-2\right)\left({ }^{\beta} \mathfrak{I}^{\alpha} \Phi^{\sigma}(x)\right)^{\frac{1}{\sigma}}\left({ }^{\beta} \mathfrak{I}^{\alpha} \Psi^{\sigma}(x)\right)^{\frac{1}{\sigma}} .
\end{aligned}
$$

Proof From the multiplication of inequalities (9) and (11), we have

$$
\left(\frac{(M+1)(m+1)}{M}\right)\left({ }^{\beta} \mathfrak{I}^{\alpha} \Phi^{\sigma}(x)\right)^{\frac{1}{\sigma}}\left({ }^{\beta} \mathfrak{I}^{\alpha} \Psi^{\sigma}(x)\right)^{\frac{1}{\sigma}} \leq\left(\left[{ }^{\beta} \mathfrak{I}^{\alpha}(\Phi(x)+\Psi(x))^{\sigma}\right]^{\frac{1}{\sigma}}\right)^{2} .
$$

Now, applying the Minkowski inequality to the right-hand side of (13), we obtain

$$
\left(\left[{ }^{\beta} \mathfrak{I}^{\alpha}(\Phi(x)+\Psi(x))^{\sigma}\right]^{\frac{1}{\sigma}}\right)^{2}
$$




$$
\begin{aligned}
& \leq\left[\left({ }^{\beta} \mathfrak{I}^{\alpha} \Phi^{\sigma}(x)\right)^{\frac{1}{\sigma}}+\left({ }^{\beta} \mathfrak{I}^{\alpha} \Psi^{\sigma}(x)\right)^{\frac{1}{\sigma}}\right]^{2} \\
& \leq\left({ }^{\beta} \mathfrak{I}^{\alpha} \Phi^{\sigma}(x)\right)^{\frac{1}{\sigma}}+\left({ }^{\beta} \mathfrak{I}^{\alpha} \Psi^{\sigma}(x)\right)^{\frac{1}{\sigma}}+2\left({ }^{\beta} \mathfrak{I}^{\alpha} \Phi^{\sigma}(x)\right)^{\frac{1}{\sigma}}\left({ }^{\beta} \mathfrak{I}^{\alpha} \Psi^{\sigma}(x)\right)^{\frac{1}{\sigma}} .
\end{aligned}
$$

Thus, from inequalities (13) and (14), we get the desired inequality (12).

Lemma 2.3 ([2]) Let $f$ be a concave function on $[a, b]$, then the following inequalities hold:

$$
f(a)+f(b) \leq f(b+a-x)+f(x) \leq 2 f\left(\frac{a+b}{2}\right) .
$$

Theorem 2.4 Let $\beta, \alpha>0, \beta \in \mathbb{C}, r, s>1$, and let $\Phi$ and $\Psi$ be two positive functions on $[0, \infty)$. If $\Phi^{r}$ and $\Psi^{s}$ are two concave functions on $[0, \infty)$, then the following inequality holds:

$$
\begin{aligned}
& 2^{-r-s}\left(\Phi(0)+\Phi\left(x^{\alpha}\right)\right)^{r}\left(\Psi(0)+\Psi\left(x^{\alpha}\right)\right)^{s}\left({ }^{\beta} \mathfrak{I}^{\alpha}\left(x^{\alpha \beta-\alpha}\right)\right)^{2} \\
& \quad \leq \mathfrak{I}^{\alpha}\left(x^{\alpha \beta-\alpha} \Phi^{r}\left(x^{\alpha}\right)\right)^{\beta} \mathfrak{I}^{\alpha}\left(x^{\alpha \beta-\alpha} \Psi^{s}\left(x^{\alpha}\right)\right) .
\end{aligned}
$$

Proof Since the functions $\Phi^{r}$ and $\Psi^{s}$ are concave on $[0, \infty)$, therefore for any $x>0, \alpha>0$ and by Lemma 2.3, we have

$$
\Phi^{r}(0)+\Phi^{r}\left(x^{\alpha}\right) \leq \Phi^{r}\left(x^{\alpha}-t^{\alpha}\right)+\Phi^{r}\left(t^{\alpha}\right) \leq 2 \Phi^{r}\left(\frac{x^{\alpha}}{2}\right)
$$

and

$$
\Psi^{s}(0)+\Psi^{s}\left(x^{\alpha}\right) \leq \Psi^{s}\left(x^{\alpha}-t^{\alpha}\right)+\Phi^{s}\left(t^{\alpha}\right) \leq 2 \Psi^{s}\left(\frac{x^{\alpha}}{2}\right)
$$

Multiplying both sides of (17) and (18) by $\frac{1}{\Gamma(\beta)}\left(\frac{x^{\alpha}-t^{\alpha}}{\alpha}\right)^{\beta-1} t^{\alpha \beta-1}, t \in(0, x)$, and integrating the resultant inequalities from 0 to $x$, we get

$$
\begin{aligned}
& \frac{\Phi^{r}(0)+\Phi^{r}\left(x^{\alpha}\right)}{\Gamma(\beta)} \int_{0}^{x}\left(\frac{x^{\alpha}-t^{\alpha}}{\alpha}\right)^{\beta-1} t^{\alpha \beta-1} d t \\
& \leq \frac{1}{\Gamma(\beta)} \int_{0}^{x}\left(\frac{x^{\alpha}-t^{\alpha}}{\alpha}\right)^{\beta-1} t^{\alpha \beta-1} \Phi^{r}\left(x^{\alpha}-t^{\alpha}\right) d t \\
& \quad+\frac{1}{\Gamma(\beta)} \int_{0}^{x}\left(\frac{x^{\alpha}-t^{\alpha}}{\alpha}\right)^{\beta-1} t^{\alpha \beta-1} \Phi^{r}\left(t^{\alpha}\right) d t \\
& \leq \frac{2 \Phi^{r}\left(\frac{x^{\alpha}}{2}\right)}{\Gamma(\beta)} \int_{0}^{x}\left(\frac{x^{\alpha}-t^{\alpha}}{\alpha}\right)^{\beta-1} t^{\alpha \beta-1} d t
\end{aligned}
$$

and

$$
\begin{aligned}
& \frac{\Psi^{s}(0)+\Psi^{s}\left(x^{\alpha}\right)}{\Gamma(\beta)} \int_{0}^{x}\left(\frac{x^{\alpha}-t^{\alpha}}{\alpha}\right)^{\beta-1} t^{\alpha \beta-1} d t \\
& \leq \frac{1}{\Gamma(\beta)} \int_{0}^{x}\left(\frac{x^{\alpha}-t^{\alpha}}{\alpha}\right)^{\beta-1} t^{\alpha \beta-1} \Psi^{s}\left(x^{\alpha}-t^{\alpha}\right) d t
\end{aligned}
$$




$$
\begin{aligned}
& +\frac{1}{\Gamma(\beta)} \int_{0}^{x}\left(\frac{x^{\alpha}-t^{\alpha}}{\alpha}\right)^{\beta-1} t^{\alpha \beta-1} \Psi^{s}\left(t^{\alpha}\right) d t \\
\leq & \frac{2 \Psi^{s}\left(\frac{x^{\alpha}}{2}\right)}{\Gamma(\beta)} \int_{0}^{x}\left(\frac{x^{\alpha}-t^{\alpha}}{\alpha}\right)^{\beta-1} t^{\alpha \beta-1} d t .
\end{aligned}
$$

Taking $x^{\alpha}-t^{\alpha}=y^{\alpha}$, we have

$$
\frac{1}{\Gamma(\beta)} \int_{0}^{x}\left(\frac{x^{\alpha}-t^{\alpha}}{\alpha}\right)^{\beta-1} t^{\alpha \beta-1} \Phi^{r}\left(x^{\alpha}-t^{\alpha}\right) d t={ }^{\beta} \mathfrak{I}^{\alpha}\left(x^{\alpha \beta-\alpha} \Phi^{r}\left(x^{\alpha}\right)\right)
$$

and

$$
\frac{1}{\Gamma(\beta)} \int_{0}^{x}\left(\frac{x^{\alpha}-t^{\alpha}}{\alpha}\right)^{\beta-1} t^{\alpha \beta-1} \Psi^{s}\left(x^{\alpha}-t^{\alpha}\right) d t={ }^{\beta} \mathfrak{I}^{\alpha}\left(x^{\alpha \beta-\alpha} \Psi^{s}\left(x^{\alpha}\right)\right) .
$$

Thus the use of (19) and (21) yields

$$
\begin{aligned}
& \left(\Phi^{r}(0)+\Phi^{r}\left(x^{\alpha}\right)\right)\left({ }^{\beta} \mathfrak{I}^{\alpha}\left(x^{\alpha \beta-\alpha}\right)\right) \\
& \quad \leq 2\left({ }^{\beta} \mathfrak{I}^{\alpha}\left(x^{\alpha \beta-\alpha} \Phi^{r}\left(x^{\alpha}\right)\right)\right) \leq 2 \Phi^{r}\left(\frac{x^{\alpha}}{2}\right)\left(\mathfrak{I}^{\alpha}\left(x^{\alpha \beta-\alpha}\right)\right) .
\end{aligned}
$$

Similarly, the use of (20) and (22) yields

$$
\begin{aligned}
\left(\Psi^{s}(0)+\Psi^{s}\left(x^{\alpha}\right)\right)\left({ }^{\beta} \mathfrak{I}^{\alpha}\left(x^{\alpha \beta-\alpha}\right)\right) & \leq 2\left({ }^{\beta} \mathfrak{I}^{\alpha}\left(x^{\alpha \beta-\alpha} \Psi^{s}\left(x^{\alpha}\right)\right)\right) \\
& \leq 2 \Psi^{s}\left(\frac{x^{\alpha}}{2}\right)\left({ }^{\beta} \mathfrak{I}^{\alpha}\left(x^{\alpha \beta-\alpha}\right)\right)
\end{aligned}
$$

From inequalities (23) and (24), it follows that

$$
\begin{gathered}
\left(\Phi^{r}(0)+\Phi^{r}\left(x^{\alpha}\right)\right)\left(\Psi^{s}(0)+\Psi^{s}\left(x^{\alpha}\right)\right)\left({ }^{\beta} \mathfrak{I}^{\alpha}\left(x^{\alpha \beta-\alpha}\right)\right)^{2} \\
\quad \leq 4\left({ }^{\beta} \mathfrak{I}^{\alpha}\left(x^{\alpha \beta-\alpha} \Phi^{s}\left(x^{\alpha}\right)\right)\right)\left({ }^{\beta} \mathfrak{I}^{\alpha}\left(x^{\alpha \beta-\alpha} \Psi^{s}\left(x^{\alpha}\right)\right)\right) .
\end{gathered}
$$

Since $\Phi$ and $\Psi$ are positive functions, therefore for any $x>0, \alpha>0, r \geq 1$, and $s \geq 1$, we have

$$
\left(\frac{\Phi^{r}(0)+\Phi^{r}\left(x^{\alpha}\right)}{2}\right)^{\frac{1}{r}} \geq 2^{-1}\left(\Phi(0)+\Phi\left(x^{\alpha}\right)\right)
$$

and

$$
\left(\frac{\Psi^{s}(0)+\Psi^{s}\left(x^{\alpha}\right)}{2}\right)^{\frac{1}{s}} \geq 2^{-1}\left(\Psi(0)+\Psi\left(x^{\alpha}\right)\right)
$$

Hence, it follows that

$$
\left(\frac{\Phi^{r}(0)+\Phi^{r}\left(x^{\alpha}\right)}{2}\right)\left({ }^{\beta} \mathfrak{I}^{\alpha}\left(t^{\alpha \beta-\alpha}\right)\right) \geq 2^{-r}\left(\Phi(0)+\Phi\left(x^{\alpha}\right)\right)^{r}\left({ }^{\beta} \mathfrak{I}^{\alpha}\left(t^{\alpha \beta-\alpha}\right)\right)
$$


and

$$
\left(\frac{\Psi^{s}(0)+\Psi^{s}\left(x^{\alpha}\right)}{2}\right)\left({ }^{\beta} \mathfrak{I}^{\alpha}\left(t^{\alpha \beta-\alpha}\right)\right) \geq 2^{-s}\left(\Psi(0)+\Psi\left(x^{\alpha}\right)\right)^{s}\left({ }^{\beta} \mathfrak{I}^{\alpha}\left(t^{\alpha \beta-\alpha}\right)\right) .
$$

From inequalities (28) and (29), we obtain

$$
\begin{aligned}
& \frac{\left(\Phi^{r}(0)+\Phi^{r}\left(x^{\alpha}\right)\right)\left(\Psi^{s}(0)+\Psi^{s}\left(x^{\alpha}\right)\right)}{4}\left({ }^{\beta} \mathfrak{I}^{\alpha}\left(t^{\alpha \beta-\alpha}\right)\right)^{2} \\
& \quad \geq 2^{-r-s}\left(\Phi(0)+\Phi\left(x^{\alpha}\right)\right)^{r}\left(\Psi(0)+\Psi\left(x^{\alpha}\right)\right)^{s}\left({ }^{\beta} \mathfrak{I}^{\alpha}\left(t^{\alpha \beta-\alpha}\right)\right)^{2} .
\end{aligned}
$$

Thus, by combining (25) and (30), we get the desired result.

Theorem 2.5 Let $\beta, \mu, \alpha>0, \beta, \mu \in \mathbb{C}, r>1, s>1$, and let $\Phi, \Psi$ be two positive functions on $[0, \infty)$. If $\Phi^{r}$ and $\Psi^{s}$ are two concave functions on $[0, \infty)$, then we have the following inequality:

$$
\begin{aligned}
2^{2-r-s} & (\Phi(0)+\Phi(x))^{r}(\Psi(0)+\Psi(x))^{s}\left({ }^{\beta} \mathfrak{I}^{\alpha}\left(x^{\alpha \mu-\alpha}\right)\right)^{2} \\
\leq & {\left[\frac{\Gamma(\mu)}{\Gamma(\beta)} \mu \mathfrak{I}^{\alpha}\left(x^{\alpha \beta-\alpha} \Phi^{r}\left(x^{\alpha}\right)\right)+{ }^{\beta} \mathfrak{I}^{\alpha}\left(x^{\mu \alpha-\alpha} \Phi^{r}\left(x^{\alpha}\right)\right)\right] } \\
& \times\left[\frac{\Gamma(\mu)}{\Gamma(\beta)} \mu \mathfrak{I}^{\alpha}\left(x^{\alpha \beta-\alpha} \Psi^{s}\left(x^{\alpha}\right)\right)+{ }^{\beta} \mathfrak{I}^{\alpha}\left(x^{\mu \alpha-\alpha} \Psi^{s}\left(x^{\alpha}\right)\right)\right] .
\end{aligned}
$$

Proof Multiplying both sides of inequalities (17) and (18) by $\frac{1}{\Gamma(\beta)}\left(\frac{x^{\alpha}-t^{\alpha}}{\alpha}\right)^{\beta-1} t^{\mu \alpha-1}, t \in(0, x)$ and then integrating the resultant inequalities with respect to $t$ from 0 to $x$, we have

$$
\begin{aligned}
& \frac{\Phi^{r}(0)+\Phi^{r}\left(x^{\alpha}\right)}{\Gamma(\beta)} \int_{0}^{x}\left(\frac{x^{\alpha}-t^{\alpha}}{\alpha}\right)^{\beta-1} t^{\mu \alpha-1} d t \\
& \leq \frac{1}{\Gamma(\beta)} \int_{0}^{x}\left(\frac{x^{\alpha}-t^{\alpha}}{\alpha}\right)^{\beta-1} t^{\mu \alpha-1} \Phi^{r}\left(x^{\alpha}-t^{\alpha}\right) d t \\
& \quad+\frac{1}{\Gamma(\beta)} \int_{0}^{x}\left(\frac{x^{\alpha}-t^{\alpha}}{\alpha}\right)^{\beta-1} t^{\mu \alpha-1} \Phi^{r}\left(t^{\alpha}\right) d t \\
& \leq \frac{2 \Phi^{r}\left(\frac{x^{\alpha}}{2}\right)}{\Gamma(\beta)} \int_{0}^{x}\left(\frac{x^{\alpha}-t^{\alpha}}{\alpha}\right)^{\beta-1} t^{\mu \alpha-1} d t
\end{aligned}
$$

and

$$
\begin{aligned}
& \frac{\Psi^{s}(0)+\Psi^{s}\left(x^{\alpha}\right)}{\Gamma(\beta)} \int_{0}^{x}\left(\frac{x^{\alpha}-t^{\alpha}}{\alpha}\right)^{\beta-1} t^{\mu \alpha-1} d t \\
& \leq \frac{1}{\Gamma(\beta)} \int_{0}^{x}\left(\frac{x^{\alpha}-t^{\alpha}}{\alpha}\right)^{\beta-1} t^{\mu \alpha-1} \Psi^{s}\left(x^{\alpha}-t^{\alpha}\right) d t \\
& \quad+\frac{1}{\Gamma(\beta)} \int_{0}^{x}\left(\frac{x^{\alpha}-t^{\alpha}}{\alpha}\right)^{\beta-1} t^{\mu \alpha-1} \Psi^{s}\left(t^{\alpha}\right) d t \\
& \leq \frac{2 \Psi^{s}\left(\frac{x^{\alpha}}{2}\right)}{\Gamma(\beta)} \int_{0}^{x}\left(\frac{x^{\alpha}-t^{\alpha}}{\alpha}\right)^{\beta-1} t^{\mu \alpha-1} d t .
\end{aligned}
$$


Now, using $x^{\alpha}-t^{\alpha}=y^{\alpha}$, we have

$$
\frac{1}{\Gamma(\beta)} \int_{0}^{x}\left(\frac{x^{\alpha}-t^{\alpha}}{\alpha}\right)^{\beta-1} t^{\mu \alpha-1} \Phi^{r}\left(x^{\alpha}-t^{\alpha}\right) d t=\frac{\Gamma(\mu)}{\Gamma(\beta)} \mu \mathfrak{I}^{\alpha}\left(x^{\alpha \beta-\alpha} \Phi^{r}\left(x^{\alpha}\right)\right)
$$

and

$$
\frac{1}{\Gamma(\beta)} \int_{0}^{x}\left(\frac{x^{\alpha}-t^{\alpha}}{\alpha}\right)^{\beta-1} t^{\mu \alpha-1} \Psi^{s}\left(x^{\alpha}-t^{\alpha}\right) d t=\frac{\Gamma(\mu)}{\Gamma(\beta)} \mu \mathfrak{I}^{\alpha}\left(x^{\alpha \beta-\alpha} \Psi^{s}\left(x^{\alpha}\right)\right) .
$$

Thus, from (32) and (34), we can write

$$
\begin{aligned}
\left(\Phi^{r}(0)+\Phi^{r}\left(x^{\alpha}\right)\right)^{\beta} \mathfrak{I}^{\alpha}\left(x^{\alpha \mu-\alpha}\right) & \leq \frac{\Gamma(\mu)}{\Gamma(\beta)} \mu \mathfrak{I}^{\alpha}\left(x^{\alpha \beta-\alpha} \Phi^{r}\left(x^{\alpha}\right)\right)+{ }^{\beta} \mathfrak{I}^{\alpha}\left(x^{\alpha \mu-\alpha} \Phi^{r}\left(x^{\alpha}\right)\right) \\
& \leq 2 \Phi^{r}\left(\frac{x^{\alpha}}{2}\right)^{\beta} \mathfrak{I}^{\alpha}\left(x^{\alpha \mu-\alpha}\right) .
\end{aligned}
$$

Similarly, from inequalities (33) and (35), we obtain

$$
\begin{aligned}
\left(\Psi^{s}(0)+\Psi^{s}\left(x^{\alpha}\right)\right)^{\beta} \mathfrak{I}^{\alpha}\left(x^{\alpha \mu-\alpha}\right) & \leq \frac{\Gamma(\mu)}{\Gamma(\beta)} \mu \mathfrak{I}^{\alpha}\left(x^{\alpha \beta-\alpha} \Psi^{s}\left(x^{\alpha}\right)\right)+{ }^{\beta} \mathfrak{I}^{\alpha}\left(x^{\alpha \mu-\alpha} \Psi^{s}\left(x^{\alpha}\right)\right) \\
& \leq 2 \Psi^{s}\left(\frac{x^{\alpha}}{2}\right) \beta \mathfrak{I}^{\alpha}\left(x^{\alpha \mu-\alpha}\right) .
\end{aligned}
$$

From (36) and (37), it follows that

$$
\begin{aligned}
& \left(\Phi^{r}(0)+\Phi^{r}\left(x^{\alpha}\right)\right)\left(\Psi^{s}(0)+\Psi^{s}\left(x^{\alpha}\right)\right)\left({ }^{\beta} \mathfrak{I}^{\alpha}\left(x^{\alpha \mu-\alpha}\right)\right)^{2} \\
& \leq\left[\frac{\Gamma(\mu)}{\Gamma(\beta)} \mu \mathfrak{I}^{\alpha}\left(x^{\alpha \beta-\alpha} \Phi^{r}\left(x^{\alpha}\right)\right)+{ }^{\beta} \mathfrak{I}^{\alpha}\left(x^{\alpha \mu-\alpha} \Phi^{r}\left(x^{\alpha}\right)\right)\right] \\
& \quad \times\left[\frac{\Gamma(\mu)}{\Gamma(\beta)} \mu \mathfrak{I}^{\alpha}\left(x^{\alpha \beta-\alpha} \Psi^{s}\left(x^{\alpha}\right)\right)+{ }^{\beta} \mathfrak{I}^{\alpha}\left(x^{\alpha \mu-\alpha} \Psi^{s}\left(x^{\alpha}\right)\right)\right] .
\end{aligned}
$$

Since $\Phi$ and $\Psi$ are positive functions, therefore for any $x>0, \alpha>0, r \geq 1, s \geq 1$, we have

$$
\frac{\Phi^{r}(0)+\Phi^{r}\left(x^{\alpha}\right)}{2} \beta \mathfrak{I}^{\alpha}\left(x^{\alpha \mu-\alpha}\right) \geq 2^{-r}\left(\Phi^{r}(0)+\Phi^{r}\left(x^{\alpha}\right)\right)^{r} \beta \mathfrak{I}^{\alpha}\left(x^{\alpha \mu-\alpha}\right)
$$

and

$$
\frac{\Psi^{s}(0)+\Psi^{s}\left(x^{\alpha}\right)}{2} \beta \mathfrak{I}^{\alpha}\left(x^{\alpha \mu-\alpha}\right) \geq 2^{-s}\left(\Psi^{s}(0)+\Psi^{s}\left(x^{\alpha}\right)\right)^{s \beta} \mathfrak{I}^{\alpha}\left(x^{\alpha \mu-\alpha}\right)
$$

Thus from (39) and (40) it follows that

$$
\begin{aligned}
& \frac{\left(\Phi^{r}(0)+\Phi^{r}\left(x^{\alpha}\right)\right)\left(\Psi^{s}(0)+\Psi^{s}\left(x^{\alpha}\right)\right)}{4}\left[{ }^{\beta} \mathfrak{I}^{\alpha}\left(x^{\alpha \mu-\alpha}\right)\right]^{2} \\
& \quad \geq 2^{-r-s}\left(\Phi^{r}(0)+\Phi^{r}\left(x^{\alpha}\right)\right)^{r}\left(\Psi^{s}(0)+\Psi^{s}\left(x^{\alpha}\right)\right)^{s}\left[\mathfrak{I}^{\alpha}\left(x^{\alpha \mu-\alpha}\right)\right]^{2} .
\end{aligned}
$$

Combining inequalities (38) and (41), we get the desired proof.

Remark 1 Letting $\beta=\mu$ in Theorem 2.5, we obtain Theorem 2.4. 


\title{
3 Concluding remarks
}

In this paper, we established Minkowski and Hermite-Hadamard inequalities for conformable fractional integral operator. If we consider $\alpha=1$ throughout the paper, then the obtained results will reduce to the said inequalities obtained by Dahmani [2]. Similarly, if we consider $\alpha=\beta=1$, then all the results will lead to the classical inequalities obtained by $[1]$.

\author{
Acknowledgements \\ Not applicable \\ Funding \\ Not applicable
}

Availability of data and materials

Not applicable

Competing interests

The authors declare that they have no competing interests.

Authors' contributions

All authors contributed equally. All authors read and approved the final manuscript.

\section{Author details}

${ }^{1}$ Department of Mathematics, College of Arts and Sciences, Prince Sattam Bin Abdulaziz University, Wadi Aldawasir, Saudi Arabia. ${ }^{2}$ College of Computer and Information Sciences, Majmaah University, Al Majmaah 11952, Saudi Arabia.

${ }^{3}$ Department of Mathematics, Shaheed Benazir Bhutto University, Sheringal, Upper Dir, Pakistan.

\section{Publisher's Note}

Springer Nature remains neutral with regard to jurisdictional claims in published maps and institutional affiliations.

Received: 19 March 2019 Accepted: 7 August 2019 Published online: 19 August 2019

\section{References}

1. Bougoffa, L.: On Minkowski and Hardy integral inequality. JIPAM. J. Inequal. Pure Appl. Math. 7(2), Article ID 60 (2006)

2. Dahmani, Z.: On Minkowski and Hermite-Hadamard integral inequalities via fractional integral. Ann. Funct. Anal. 1, $51-58(2010)$

3. Dragomir, S.S., Pearce, C.E.M.: Selected topics on Hermite-Hadamard inequalities and applications, RGMIA Monographs, Victoria University, (2000). http://www.staff.vu.edu.au/RGMIA/monographs/hermite-hadamard.html

4. Guessab, A., Schmeisser, G.: Sharp integral inequalities of the Hermite-Hadamard type. J. Approx. Theory 115(2), 260-288 (2002)

5. Habib, S., Mubeen, S., Naeem, M.N.: Chebyshev type integral inequalities for generalized $k$-fractional conformable integrals. J. Inequal. Spec. Funct. 9(4), 53-65 (2018)

6. Huang, C.J., Rahman, G., Nisar, K.S., Ghaffar, A., Qi, F.: Some inequalities of the Hermite-Hadamard type for $k$-fractional conformable integrals. Aust. J. Math. Anal. Appl. 16(1) (2019)

7. Jarad, F., Uurlu, E., Abdeljawad, T., Baleanu, D.: On a new class of fractional operators. Adv. Differ. Equ. 2017(1), 247 (2017). https://doi.org/10.1186/s13662-017-1306-z

8. Mitrinovič, D.S., Lackovíc, I.B.: Hermite and convexity. Aequ. Math. 28, 229-232 (1985)

9. Mubeen, S., Habib, S., Naeem, M.N.: The Minkowski inequality involving generalized k-fractional conformable integral. J. Inequal. Appl. 2019, 81 (2019). https://doi.org/10.1186/s13660-019-2040-8

10. Özdemir, M.E., Yıldız, C., Akdemir, A.O., Set, E.: On some inequalities for s-convex functions and applications. J. Inequal. Appl. 2013, Article ID 333 (2013)

11. Qi, F., Habib, S., Mubeen, S., Naeem, M.N.: Generalized k-fractional conformable integrals and related inequalities. AIMS Ser. Appl. Math. 4(3), 343-358 (2019)

12. Qi, F., Rahman, G., Hussain, S.M., Du, W.S., Nisa, K.S.: Some inequalities of Čebyšev type for conformable $k$-fractional integral operators. Symmetry 2018(10), 614 (2018)

13. Rahman, G., Nisar, K.S., Qi, F.: Some new inequalities of the Grüss type for conformable fractional integrals. AIMS Ser. Appl. Math. 3(4), 575-583 (2018)

14. Rahman, G., Ullah, Z., Khan, A., Set, E., Nisar, K.S.: Certain Chebyshev-type inequalities involving fractional conformable integral operators. Mathematics 2019(7), 364 (2019)

15. Samko, S.G., Kilbas, A.A., Marichev, O.I.: Fractional Integrals and Derivatives: Theory and Applications. Gordon \& Breach, Reading (1993)

16. Set, E., Mumcu, I.., Özdemir, M.E.: Grüss type inequalities involving new conformable fractional integral operators. AIP Conf. Proc. 1991, Article ID 020020 (2018). https://doi.org/10.1063/1.5047893

17. Set, E., Özdemir, M.E., Sarıkaya, M.Z.: Inequalities of Hermite-Hadamard's type for functions whose derivatives absolute values are $m$-convex. AIP Conf. Proc. 1309(1), 861-873 (2010)

18. Srivastava, H.M., Choi, J.: Zeta and q-Zeta Functions and Associated Series and Integrals. Elsevier, Amsterdam (2012) 